\title{
Precision Dynamic Aperture Tracking in Rings
}

\author{
F. Méot \\ CEA DSM DAPNIA SACM, F-91191 Saclay \\ fmeot@cea.fr\}
}

\begin{abstract}
The paper presents a variety of results concerning dynamic aperture tracking studies, including most recent ones, obtained using a highly symplectic numerical method based on truncated Taylors series. Comparisons with various codes, that had to be performed in numerous occasions, are also addressed.
\end{abstract}

\section{Introduction}

Precision is a strong concern in long term multiturn particle tracking in accelerators and storage rings. Considering the dramatic speed increase of informatics means, it became evident several years ago that there were no good reasons left for keeping using simplified field models and simplistic mapping methods, that both would lead, although fast, to erroneous results as to possible effects of field non-linearities on long-term particle motion.

This has motivated upgrading of the ray-tracing code Zgoubi [1], formerly developed by J. C. Faivre and D. Garreta at Saclay for the calculating trajectories in magnetic spectrometer field maps [2]. First multiturn ray-tracing trials concerned spin tracking for the purpose of studying the installation of a partial Siberian snake in the $3 \mathrm{GeV}$ synchrotron Saturne ; the main task there was twofold, on the one hand assure symplectic transport throughout the about $10^{4}$ turn lasting depolarizing resonance crossing, on the other hand satisfy $\sqrt{S_{x}^{2}+S_{z}^{2}+S_{s}^{2}}=1$ while tracking the all three spin components $\left(S_{x}, S_{z}, S_{s}\right)$ in presence in particular of dipole and quadrupole fringe fields that have a major role in depolarization, which all proved to work quite well [3]. That led to cope with (much) larger size machines, at first reasonably close to first order behavior, and eventually including all sorts of more or less strong sources of non-linearities [4]-[8], without forgetting sophisticated non-linear motion in an electrostatic storage ring [9].

In the following, we first recall the principles of the integration method and field models used. Next we summarize some meaningful numerical results so obtained.

\section{Numerical method}

\section{$2.1 \quad$ Integration}

Zgoubi solves the Lorentz equation $d(m \boldsymbol{v}) / d t=q(\boldsymbol{E}+\boldsymbol{v} \times \boldsymbol{B})$ by stepwise Taylor expansions of the vector position $\boldsymbol{R}$ and velocity $\boldsymbol{v}$, which writes 


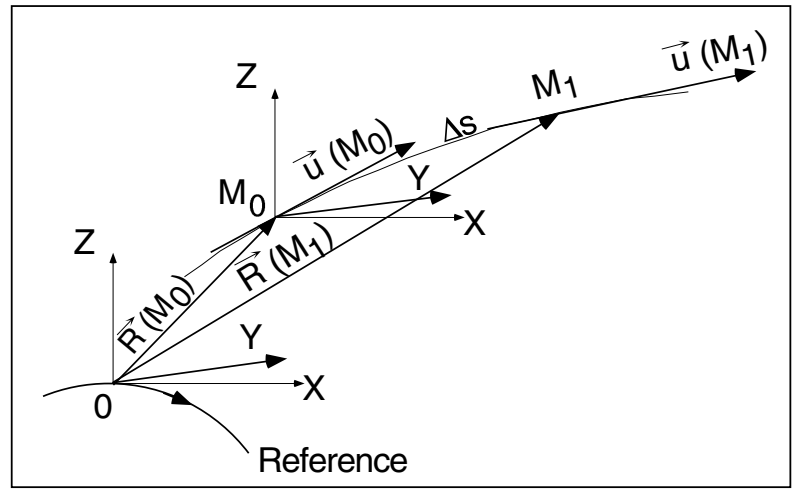

Fig. 1. Particle motion in the Zgoubi frame, and parameters used in the text.

$$
\begin{gathered}
\boldsymbol{R}\left(M_{1}\right)=\boldsymbol{R}\left(M_{0}\right)+\boldsymbol{u}\left(M_{0}\right) \Delta s+\boldsymbol{u}^{\prime}\left(M_{0}\right) \Delta s^{2} / 2 !+\ldots \\
\boldsymbol{u}\left(M_{1}\right)=\boldsymbol{u}\left(M_{0}\right)+\boldsymbol{u}^{\prime}\left(M_{0}\right) \Delta s+\boldsymbol{u}^{\prime \prime}\left(M_{0}\right) \Delta s^{2} / 2 !+\ldots
\end{gathered}
$$

wherein $\boldsymbol{u}=\boldsymbol{v} / v$ with $v=|\boldsymbol{v}|, \quad d s=v d t, \quad \boldsymbol{u}^{\prime}=d \boldsymbol{u} / d s$, and with $\quad m \boldsymbol{v}=$ $m v \boldsymbol{u}=q B \rho \boldsymbol{u}, B \rho=$ rigidity of the particle with mass $m$ and charge $q$. The derivatives $\boldsymbol{u}^{(n)}=d^{n} \boldsymbol{u} / d s^{n}$ are obtained as functions of the field derivatives $d^{n} \boldsymbol{B} / d s^{n}, d^{n} \boldsymbol{E} / d s^{n}$ by recursive differentiation of the equation of motion, in the following way.

Magnetic fields : In purely magnetic optical elements the particle rigidity is constant and the recursive differentiation simply writes $B \rho \boldsymbol{u}^{\prime}=\boldsymbol{u} \times \boldsymbol{B}, B \rho \boldsymbol{u}^{\prime \prime}=$ $\boldsymbol{u}^{\prime} \times \boldsymbol{B}+\boldsymbol{u} \times \boldsymbol{B}^{\prime}$, and so forth.

Electrostatic fields : In purely electric fields the rigidity varies and the recursive differentiation takes the less simple form $(B \rho)^{\prime} \boldsymbol{u}+B \rho \boldsymbol{u}^{\prime}=\boldsymbol{E} / v,(B \rho)^{\prime \prime} \boldsymbol{u}+$ $2(B \rho)^{\prime} \boldsymbol{u}^{\prime}+B \rho \boldsymbol{u}^{\prime \prime}=(1 / v)^{\prime} \boldsymbol{E}+\boldsymbol{E}^{\prime} / v$, etc., whereas the rigidity itself is also obtained by Taylor expansion

$$
(B \rho)\left(M_{1}\right)=(B \rho)\left(M_{0}\right)+(B \rho)^{\prime}\left(M_{0}\right) \Delta s+(B \rho)^{\prime \prime}\left(M_{0}\right) \Delta s^{2} / 2 !+\ldots
$$

The derivatives $(B \rho)^{(n)}=d^{n}(B \rho) / d s^{n}$ are in turn obtained by alternate recursive differentiation of, on the one hand $(B \rho)^{\prime}=(\boldsymbol{e} \cdot \boldsymbol{u}) / v$, and on the other hand $B \rho(1 / v)^{\prime}=\left(1 / c^{2}\right)(\boldsymbol{e} \cdot \boldsymbol{u})-(1 / v)(B \rho)^{\prime}$.

By principle these transformations are symplectic, in practice the Taylor series are truncated so that best precision is obtained when the higher order derivatives in the truncated series are zero (at least to machine accuracy) .

\section{$2.2 \quad$ Field models}

The major components in accelerators, at least relevant to DA studies, are multipoles or multipolar defects. Explicit analytical expressions of multipole fields and of their derivatives are drawn from the regular 3-D scalar potential (that 
holds for both magnetic and (skew-) electric multipoles)

$$
V_{n}(s, x, z)=(n !)^{2}\left\{\sum_{q=0}^{\infty}(-)^{q} \frac{\alpha_{n, 0}^{(2 q)}(s)}{4^{q} q !(n+q) !}\left(x^{2}+z^{2}\right)^{q}\right\}\left\{\sum_{m=0}^{n} \frac{\sin \left(m \frac{\pi}{2}\right) x^{n-m} z^{m}}{m !(n-m) !}\right\}
$$

where $\mathrm{s}, \mathrm{x}, \mathrm{z}$ coordinates are respectively curvilinear, transverse horizontal and vertical, $\alpha_{n, 0}(s)$ ( $n=1,2,3$,etc.) describe the longitudinal form of the field, including end fall-offs, and $\alpha_{n, 0}^{(2 q)}=d^{2 q} \alpha_{n, 0} / d s^{2 q}$. Note that, within magnet body or as well when using hard edge field model, $d^{2 q} \alpha_{n, 0} / d s^{2 q} \equiv 0(\forall q \neq 0)$ hence the field and derivatives derive from the simplified potentials

$$
V_{1}(x, z)=G_{1} z, \quad V_{2}(x, z)=G_{2} x z, \quad V_{3}(x, z)=G_{3}\left(x^{2}-z^{2} / 3\right) z, \quad \text { etc. }
$$

where $G_{n} / B \rho$ is the strength.

Field fall-off at magnet ends : As to the field fall-off on axis at magnet ends orthogonally to the effective field boundary (EFB), it is modeled by (after Ref. [10, page 240])

$$
\alpha_{n, 0}(d)=\frac{G_{n}}{1+\exp [P(d)]} \text { with } P(d)=C_{0}+C_{1} \frac{d}{\lambda_{n}}+C_{2}\left(\frac{d}{\lambda_{n}}\right)^{2}+\ldots+C_{5}\left(\frac{d}{\lambda_{n}}\right)^{5}
$$

where $d$ is the distance to the EFB and coefficients $\lambda_{n}, C_{0}-C_{5}$ can be determined from prior matching with realistic numerical fringe field data.

\section{More fields}

Zgoubi is actually a genuine compendium of optical elements of all sorts, magnetic and/or electric, with fields derived from more or less sophisticated analytical models as above. This allows simulating with precision regular rings.

\section{DA tracking}

In the following various results drawn from (unpublished) reports are presented, with the aim of showing the accuracy and effectiveness of the ray-tracing method.

\subsection{Effect of $b_{10}$ in low- $\beta$ quadrupoles in LHC [5]}

The multipole defect $b_{10}$ in LHC low- $\beta$ quadrupoles derives from (Eq. 1, Fig. 2)

$$
V_{10}(s, x, z) \approx\left(\alpha_{10,0}-\frac{\alpha_{10,0}^{\prime \prime}}{44}\left(x^{2}+z^{2}\right)\right)\left(10 x^{8}-120 x^{6} z^{2}+252 x^{4} z^{4}-120 x^{2} z^{6}+10 z^{8}\right)
$$

The goal in this problem was to assess the importance of the way $b_{10}$ is distributed along the quadrupole. Three models are investigated (Fig. 2) : hard edge (a), a regular smooth fall-off at quadrupole ends (b), a lump model in 


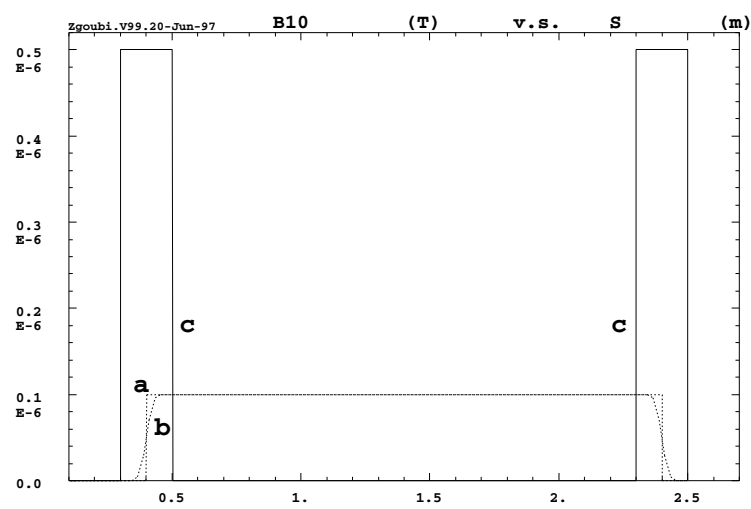

Fig. 2. Fringe field models used for assessing effect of $b_{10}$ error on particle dynamics.

which $b_{10}$ is zero in the body and the integral strength is shared between the two ends (c). In all three cases the overall field integral is the same.

Optical aberrations at IP 5 : It can be seen from Fig. 3 that $b_{10}=-0.00510^{-4}$ strongly distorts the aberration curves that would otherwise show a smooth, cubic shape. The aberration is of the form $x_{I P} \approx\left(\frac{x}{x^{\prime 3}}\right) x^{\prime^{3}}+\left(\frac{x}{x^{\prime 9}}\right) x_{0}^{\prime 9}$ with $x_{0}^{\prime}$ being the starting angle at point-to-point imaging location upstream of the interaction point (IP). The coefficient $\left(x / x^{\prime 3}\right)$ is mostly due to geometrical errors introduced

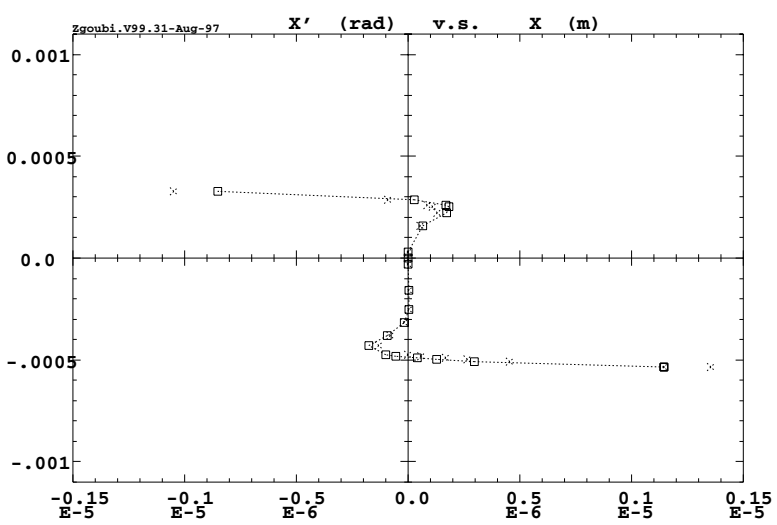

Fig. 3. Optical aberrations with inclined closed orbit at IP5 $(0.1 \sqrt{2} \mathrm{mrad}$ c.o. angle inclined $45^{\circ}$ ) ; fringe fields are set in separation dipoles D1/D2 and in the quadrupoles for the main component $b_{2}$. Squares : hard edge or fringe field model. Crosses : lump $b_{10}$ model.

by the quadrupole and $\left(x / x^{\prime 9}\right)$ is due to $b_{10}$; they have opposite signs and therefore act in opposite ways. The turn-round region between the two effects 
gets closer to the $\mathrm{x}$-axis the stronger $b_{10}$. In particular with the present value of $b_{10}$ a $\pm 1 \mu \mathrm{m}$ extent at the image is reached with starting angle within -10 to $15 \sigma_{x_{0}^{\prime}}$, about twice smaller than without $b_{10}$.

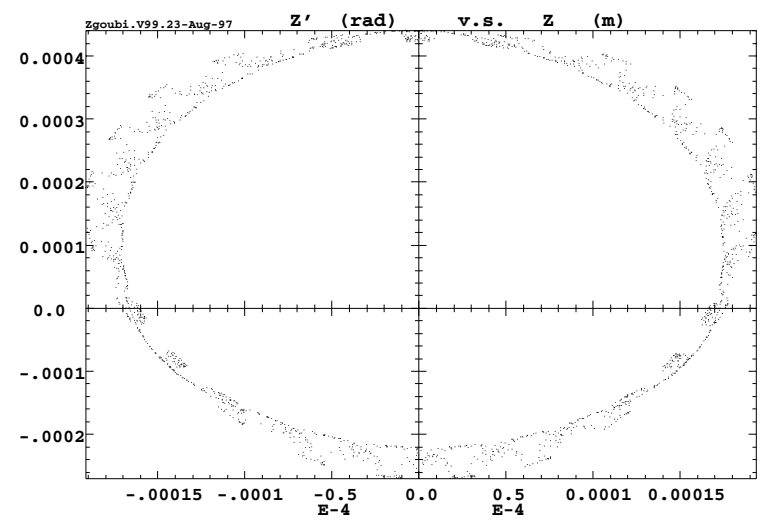

Fig. 4. Vertical phase space plot of a particle launched with $\mathrm{x}=\mathrm{x}=\mathrm{y}=0$ and $y^{\prime}=$ $11.0 \sigma$, in presence of inclined $0.28 \mathrm{mrad}$ c.o. angles of identical signs at IP1 and IP5 simultaneously, with lumped $b_{10}$ model (longitudinal distribution 'c' in Fig. 2).

DA tracking : Multiturn tracking of the dynamic aperture must stand the comparison. At first sight, considering the violent turn-round in the aberration curves (Fig. 3) and the fact that it occurs at $x_{0}^{\prime} \approx 9.5 \sigma_{x^{\prime}}$ whatever the longitudinal model for $b_{10}$, it can be expected that, on the one hand all three models provide similar DA, on the other hand the DA be about $9.5 \sigma$ as well. This has been precisely confirmed by DA tracking, details can be found in Ref. [5]. As an illustration Fig. (4) provides a sample transverse phase space at 9.5 $\sigma$ DA.

\subsection{Fringe field effects in the Fermilab $50 \mathrm{GeV}$ muon storage ring [7]}

The goal here was to examine possible effects of quadrupole fringe fields (Fig. 5) in the FERMILAB $50 \mathrm{GeV}$ muon storage ring Feasibility I Study. An interesting outcome - amongst others - is a clear disagreement with similar studies subject to earlier publication.

Table 1 recalls the main machine parameters. Unprecedented apertures are required in the muon storage ring for a Neutrino Factory because of the exceptionally large emittances associated with the intense muon beams which must be accepted. The superconducting arc quadrupoles require a $14 \mathrm{~cm}$ bore, $3.6 \mathrm{~T}$ poletip field, which leads to strong, extended fringe fields.

Large acceptance motion in the absence of fringe fields is shown in Fig. 6 . Particles are launched for a few hundred turns ray-tracing with initial coordinates either $x_{0}=1-4 \times 10^{-2} \mathrm{~m} \approx 4 \sigma_{x}$ and $\epsilon_{z}=0$ (left column in the figure), or 

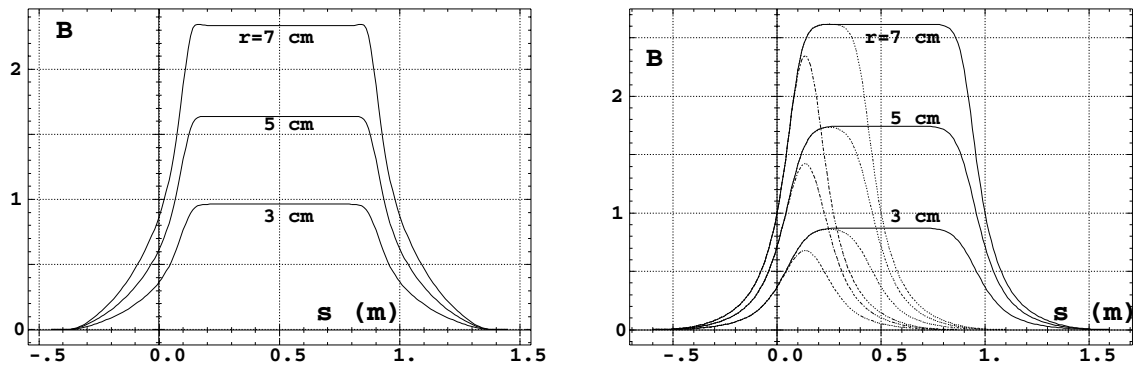

Fig. 5. Shape of the magnetic field $B(s)$ (arbitrary units) observed 3,5 or $7 \times 10^{-2} \mathrm{~m}$ off-axis along the quadrupoles. Left : arc quadrupole (QF1, QD1 families) including sextupole component. Right : $1 \mathrm{~m}, 0.5 \mathrm{~m}$ or $0.27 \mathrm{~m}$ long matching quadrupoles.
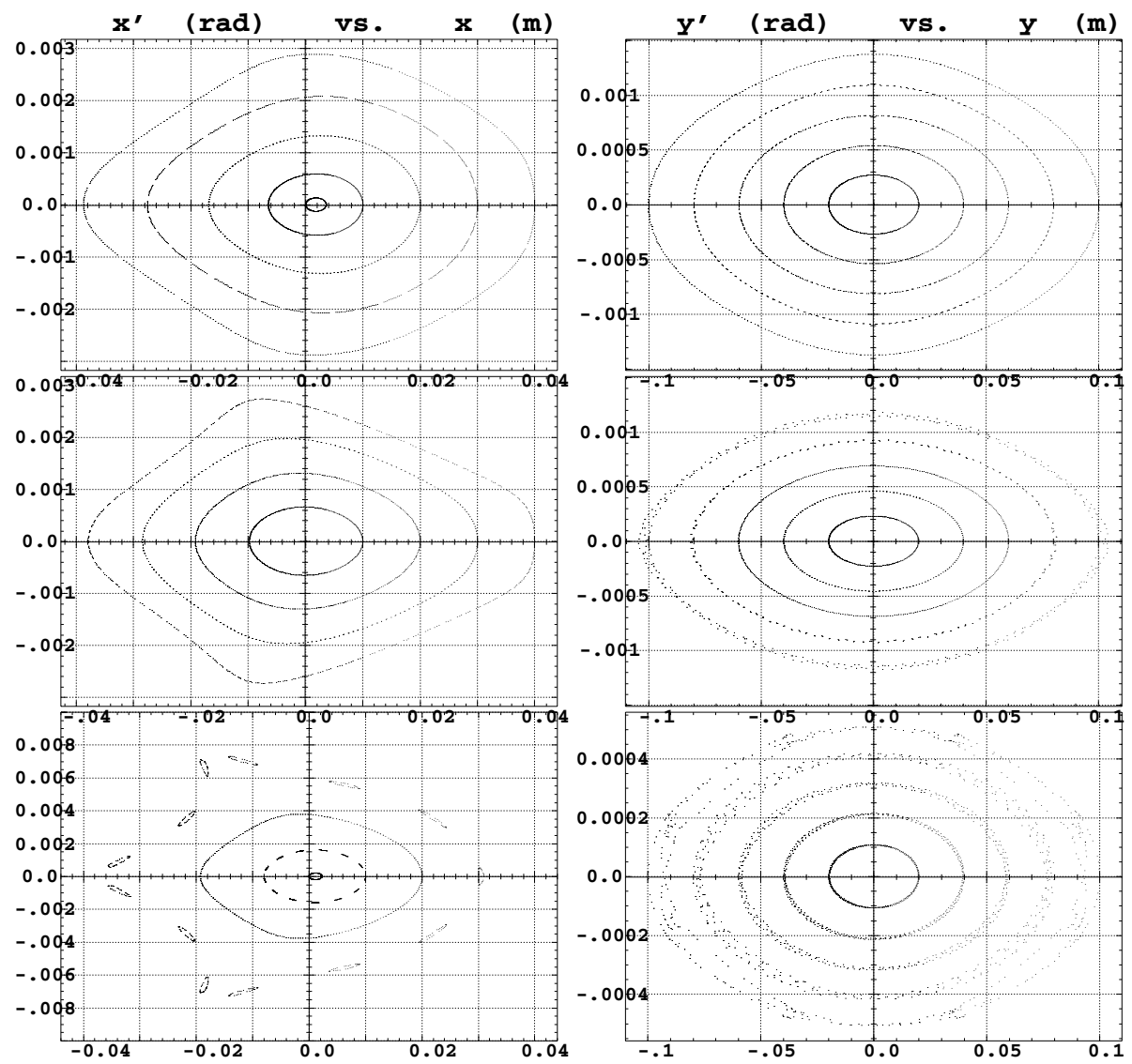

Fig. 6. Phase space plots up to DA region, no fringe fields, sextupoles are on. All particles survive except the largest amplitude one in the bottom left-hand plot (with initial conditions $x_{0}=0.04 \mathrm{~m} \approx 4 \sigma_{x}, \delta p / p=-2 \%$ ) 
Table 1. Storage Ring Parameters at $50-\mathrm{GeV}$

$\begin{array}{lcc}\text { Circumference } & \mathrm{m} & 1752.8 \\ \text { matching and dispersion suppression } & \mathrm{m} & 44.1 \\ \text { High- } \beta \text { FODO straight } & \mathrm{m} & 688 \\ \beta_{x \max } / \beta_{z \max } & \mathrm{m} & 435 / 484 \\ \nu_{x} / \nu_{z} & 13.63 / 13.31 \\ \text { natural chromaticity } & -23.9 /-23.9 \\ d \nu_{x} / d \frac{\epsilon_{x}}{\pi} / d \nu_{z} / d \frac{\epsilon_{z}}{\pi} \text { (sextus on) } & -3.8 /-11\end{array}$

$z_{0}=1-10 \times 10^{-2} \mathrm{~m} \approx 4 \sigma_{z}$ and $\epsilon_{x}=0$ (right col.), and with, top row $:+2 \%$ offmomentum ; middle : on-momentum ; bottom row : $-2 \%$ off-momentum. Setting the fringe fields leaves Fig. 6 practically unchanged [7], which is a good indication of the strong symplecticity of the method, considering the strong non-linearities so introduced (Fig. 5). It was therefore concluded to their quasi-innocuousness, contrary to Ref. [12] whose questionable results probably come from its using too low order mapping [7].

\section{An electrostatic storage ring}

This design study concerned a low energy electrostatic storage ring employed as a multiturn time of flight mass spectrometer (TOFMS), the principle being that multiturn storage is a convenient way to reach high resolution mass separation allied with small size apparatus. The ring is built from electrostatic parallel-plate mirrors which are highly non-linear optical elements, used for both focusing and bending.

Fig. 7 shows the geometry of the periodicity-2 ring of concern, built from the symmetric cell (LV, LH, MA, MB, LH, LV) wherein MA and MB are 3-electrode parallel-plate mirrors acting as 90 degrees deflector (i.e., bends), LV and LH are similar devices used as transmission vertical and horizontal lenses. The potential experienced by particles in these lenses and mirrors writes

$$
V(X, Z)=\sum_{i=2}^{3} \frac{V_{i}-V_{i-1}}{\pi} \arctan \frac{\sinh \left(\pi\left(X-X_{i-1}\right) / D\right)}{\cos (\pi Z / D)}
$$

( $V i$ are the potentials at the 3 electrode pairs, $X i$ are the locations of the slits, $X$ is the distance from the origin taken at the first slit, $D$ is the inter-plate distance). In terms of non-linearities, a study of the second order coefficients of the cell reveals significant $\mathrm{x}-\mathrm{z}$, as well as $\mathrm{z}-\delta p / p$ coupling. In addition particles are slowed down by the mirror effect so that their rigidity $B \rho$ undergoes dramatic variations (hence strong coefficients in its Taylor series).

A good indication of symplectic integration here is obtained by checking easily accessed constants, such as total energy (sum of the local kinetic and and potential $(\mathrm{V}(\mathrm{X}, \mathrm{Y}, \mathrm{Z}))$ energies), kinetic momentum, or else, calculated from 


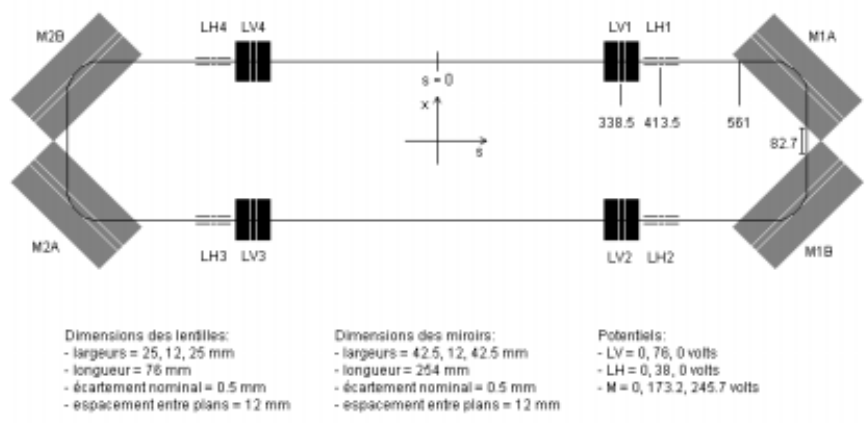

Fig. 7. The TOFMS ring. Quotations are in millimeters.

position $R(x, z, s)$ and speed $u(x, z, s)$. Such ckecks in particular led to the appropriate minimum value of the integration step $\Delta s$ in the mirror bends.

DA tracking trials are shown in Fig. 9 for various potential values at LV. They were realized in order to assess the ring acceptance and prove to be well behaved, in particular free of any sign of spiral motion, in spite of the strongly non-linear fields. The horizontal acceptance comes out to be rather large, $\pm 8 \mathrm{~mm}$ as observed at $s=0$, whereas the vertical one is drastically affected by the nonlinearities and does not exceed $\pm 1 \mathrm{~mm}$.

\section{Conclusion}

The Zgoubi ray-tracing method has been described, examples of its use have been given and show its very high accuracy that makes it an efficient tool for precision DA tracking.

\section{References}

1. The ray-tracing code Zgoubi, F. Méot, CERN SL/94-82 (AP) (1994), NIM A 427 (1999) 353-356.

Zgoubi users' guide, F. Méot and S. Valero, Report CEA DSM/DAPNIA/SEA/9713, and FERMILAB-TM-2010, Dec. 1997.

2. See for instance, Le spectromètre II, J. Thirion et P. Birien, Internal Report DPhN/ME, CEA Saclay, 23 Déc. 1975.

3. A numerical method for combined spin tracking and ray-tracing of charged particles, F. Méot, NIM A 313 (1992) 492, and Proc. EPAC Conf. (1992) p.747.

4. On the effects of fringe fields in the LHC ring, F. Méot, Part. Acc., 1996, Vol. 55, pp.83-92.

5. Concerning effects of fringe fields and longitudinal distribution of $b_{10}$ in low- $\beta$ regions on dynamics in LHC, F. Méot and A. Parîs, Report FERMILAB-TM-2017, Aug. 1997. 


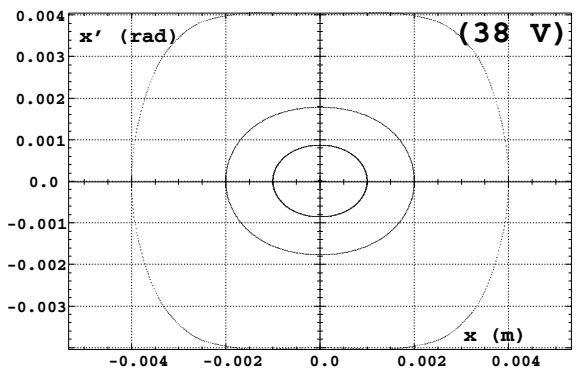

Fig. 8. 1000-turn horizontal acceptance as observed at $s=0$, with $V_{L H}=38$ Volts, $V_{L V}=76$ Volts.
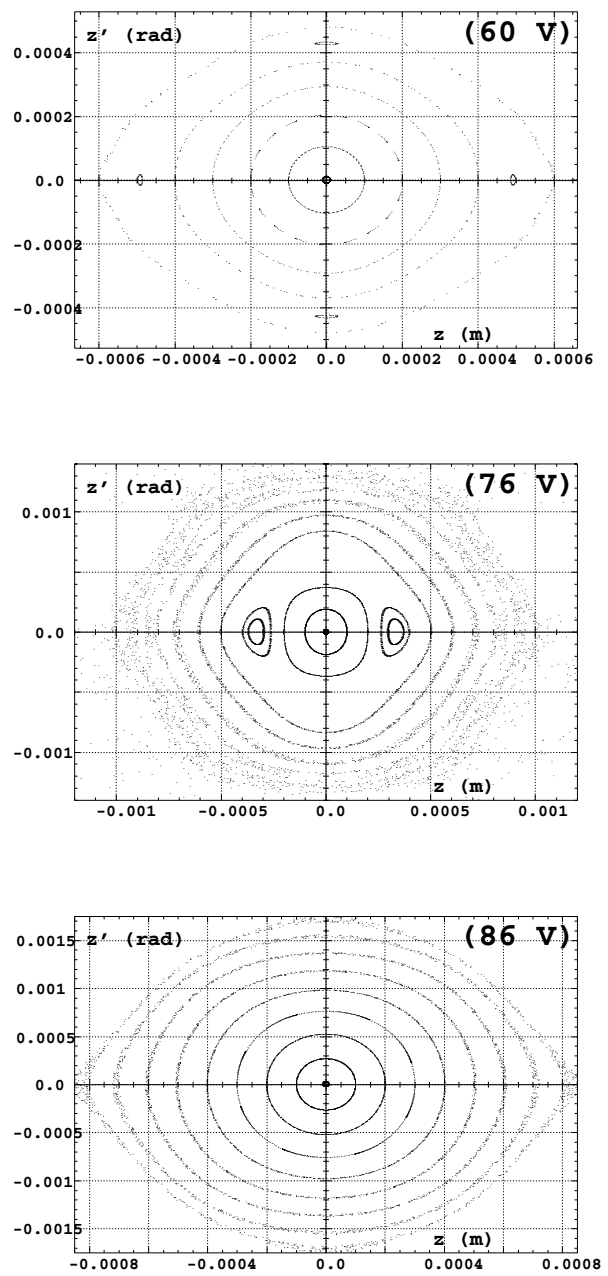

Fig. 9. 1000-turn vertical acceptance as observed at $s=0$, for $V_{L V}=$ 60,76 and 86 Volts. 
6. On fringe field effects in the CERN $50 \mathrm{GeV}$ muon storage ring, F. Méot, Internal Report CEA DSM DAPNIA/SEA-00-02, Saclay (2000).

7. On fringe field effects in the FERMILAB $50 \mathrm{GeV}$ muon storage ring, C. Johnstone and F. Méot, Internal Report CEA DSM DAPNIA/SEA-01-05, Saclay (2001) and Proc. PAC 01 Conf. (2001).

8. DA studies in the FERMILAB proton driver, F. Méot, C. Johnstone, A. Drozhdin, Internal Report CEA DSM DAPNIA/SEA-01-05, Saclay (2001).

9. Multiturn ray-tracing based design study of a compact time of flight mass spectrometer ring, M. Baril, F. Méot, D. Michaud, Proc. ICAP2000 Conf., Darmstadt, Germany, 11-14 Sept. 2000.

10. Deflecting magnets, H.A. Enge, in Focusing of charged particles, volume 2, A. Septier ed., Academic Press, New-York and London (1967).

11. A. Faus-Golfe and A. Verdier, Dynamic aperture limitations of the LHC in physics conditions due to low- $\beta$ insertions, Proc. EPAC Conf. 1996.

12. Fringe fields and dynamic aperture in the FNAL muon storage ring, F. Zimmermann et als., CERN-SL-2000-011 AP (May 4, 2000). 